\title{
Pathogenic Mycobacterium bovis strains differ in their ability to modulate the proinflammatory activation phenotype of macrophages
}

Marcelle RM Andrade ${ }^{1}$, Eduardo P Amaral ${ }^{2}$, Simone CM Ribeiro ${ }^{1}$, Fabricio M Almeida', Tanara V Peres', Verônica Lanes ${ }^{1}$, Maria Regina D'Império-Lima² and Elena B Lasunskaia ${ }^{1 *}$

\begin{abstract}
Background: Tuberculosis, caused by Mycobacterium tuberculosis or Mycobacterium bovis, remains one of the leading infectious diseases worldwide. The ability of mycobacteria to rapidly grow in host macrophages is a factor contributing to enhanced virulence of the bacteria and disease progression. Bactericidal functions of phagocytes are strictly dependent on activation status of these cells, regulated by the infecting agent and cytokines. Pathogenic mycobacteria can survive the hostile environment of the phagosome through interference with activation of bactericidal responses. To study the mechanisms employed by highly virulent mycobacteria to promote their intracellular survival, we investigated modulating effects of two pathogenic $M$. bovis isolates and a reference $M$. tuberculosis H37Rv strain, differing in their ability to multiply in macrophages, on activation phenotypes of the cells primed with major cytokines regulating proinflammatory macrophage activity.

Results: Bone marrow- derived macrophages obtained from C57BL/6 mice were infected by mycobacteria after a period of cell incubation with or without treatment with IFN- $\gamma$, inducing proinflammatory type-1 macrophages (M1), or IL-10, inducing anti-inflammatory type-2 cells (M2). Phenotypic profiling of M1 and M2 was then evaluated. The M. bovis strain MP287/03 was able to grow more efficiently in the untreated macrophages, compared with the strains B2 or H37Rv. This strain induced weaker secretion of proinflammatory cytokines, coinciding with higher expression of M2 cell markers, mannose receptor (MR) and arginase-1 (Arg-1). Treatment of macrophages with IFN- $\gamma$ and infection by the strains B2 and H37Rv synergistically induced M1 polarization, leading to high levels of inducible nitric oxide synthase (iNOS) expression, and reduced expression of the Arg-1. In contrast, the cells infected with the strain MP287/03 expressed high levels of Arg-1 which competed with iNOS for the common substrate arginine, leading to lower levels of NO production.
\end{abstract}

Conclusions: The data obtained demonstrated that the strain, characterized by increased growth in macrophages, down- modulated classical macrophage activation, through induction of an atypical mixed M1/M2 phenotype.

Keywords: Bone marrow- derived macrophages, Mycobacterium bovis, Macrophage activation phenotype, Interferon- $\gamma$, IL-10

\footnotetext{
* Correspondence: elassounskaia@gmail.com

${ }^{1}$ Laboratory of Biology of Recognition, Universidade Estadual do Norte

Fluminense, Campos Rio de Janeiro 28013-602, Brazil

Full list of author information is available at the end of the article
} 


\section{Background}

Macrophages are key cells of innate immunity to different mycobacterial infections, including human and bovine tuberculosis caused predominantly by Mycobacterium tuberculosis (Mtb) and Mycobacterium bovis (Mbv), respectively. The functions of $M \Phi$ after infection with mycobacteria are strictly dependent on the activation phenotype, which is determined by bacteria- induced signaling through the pattern-recognition receptors (PRRs), leading to innate $\mathrm{M} \Phi$ activation, and is also regulated by a variety of signals from the surrounding microenvironment. The most important of these signals are cytokines produced by activated lymphocytes and other cells.

Macrophages primed with Th1 cytokine (IFN- $\gamma$ ) polarize to proinflammatory M1 cells, readily increasing the level of activation in the presence of microbial ligands, and developing the phenotype typical of classically activated macrophages, CAM [1]. These cells produce large amounts of proinflammatory cytokines and generate reactive oxygen (ROI) and nitrogen (RNI) intermediates which enhance bactericidal and cytotoxic $M \Phi$ functions. In contrast, macrophages activated with Th2 cytokines (IL-4, IL-13), exposed to immune complexes in combination with TLR ligands, or IL-10, polarize to distinct M2 phenotypes, M2a, M2b and M2c, respectively, associated with alternatively activated macrophages (AAM), which display anti-inflammatory and tissue repair activities [2]. The M2 macrophages are characterized by expression of typical markers, including increased arginase 1 (Arg-1) expression and activity, increased expression of scavenger and mannose (MR/ CD206) receptors, and production of the antiinflammatory cytokine (IL-10), which is more pronounced in the AAM induced by exogenic IL-10. The $M \Phi$ primed by IL-10 were demonstrated to secrete none, or very low levels, of proinflammatory cytokines in response to bacterial LPS, but produce antiinflammatory IL-10 and TGF- $\beta$, that prompted Gordon to term this M2 state the 'innate/acquired inactivation' [1] and include these cells to group of 'regulatory' MF [3]. The cytokines produced by Th1 and Th2 cells or IL-10 induce extreme phenotypes of $M \Phi$ activation characterized by distinct markers. After infection, microbial products can modulate $M \Phi$ activation through PRR-dependent signaling, providing a wide range of $\mathrm{M} \Phi$ phenotypes between the two extremes [4].

During acute inflammatory responses to Mtb, macrophages are typically polarized to M1 under the effects of mycobacterial agonists for PRRs and IFN- $\gamma$ produced by Th1, and exert potent anti-microbial effects [5]. The transcriptomic analysis of responses of murine bone marrow- derived macrophages (BMDM) to Mtb and IFN- $\gamma$ revealed an overlap of genes modulated by mycobacteria and IFN $-\gamma$, which corresponded to a M1 profile [6,7]. In contrast, pretreatment of the BMDM with IL-4 resulted in the M2 transcriptional profile, and these cells presented delayed, and partially diminished, anti-mycobacterial responses [7]. These data were obtained employing the 'laboratory' Mtb strain H37Rv, widely used as a reference virulent strain for studies of tuberculosis pathogenesis. However, there is mounting evidence that strains of Mtb and Mbv circulating in human and animal populations are more genetically and functionally diverse than previously appreciated, demonstrating strain-dependent variation in virulence [8-11]. In the model of $M \Phi$ infection, highly virulent and epidemiologically successful strains of Mtb were able to grow faster than the less virulent isolates [12,13]. The enhanced bacterial growth was observed not only in the intact murine $M \Phi$, but also in those primed by IFN $-\gamma$ $[14,15]$, suggesting, that at least some virulent strains of Mtb were able to inhibit CAM. Additionally, highly virulent Mtb were able to switch the initial Th1-type reaction, associated with high levels of IFN- $\gamma$ production in the infected mice, to potent Treg cell response leading to production of IL-10, which reduced the bactericidal activities of $M \Phi$ [11]. In contrast to Mtb, modulating effects of pathogenic Mbv strains, differing in virulenceassociated properties, on the $M \Phi$ activation phenotypes, determined by main regulating cytokines, IFN- $\gamma$ and IL10 , have not been yet elucidated.

In this work, we studied the effects of pathogenic Mbv isolates and reference Mtb strain H37Rv, differing in their ability to grow intracellularly in murine $M \Phi$, on polarization of these cells to M1 and M2 phenotypes induced by the treatment with IFN- $\gamma$ and IL-10, respectively. Expression levels of typical M1 and M2 markers were evaluated. Additionally, we verified intracellular signaling pathways that could regulate production of microbicidal RNIs, through the modulation of iNOS and Arg-1 expression.

Our results demonstrated that the Mbv strain MP287/ 03, characterized by increased intracellular survival and growth, in contrast to other strains, inhibited classical $M \Phi$ activation, switching the M1 activation profile of the cells, stimulated with IFN- $\gamma$, to a mixed M1/M2 phenotype. Increased expression of Arg-1, observed in these cells, coincided with low levels of nitric oxide production, suggesting that reduced exposure of bacteria to nitrosative stress contributed to increased intracellular survival of these bacteria.

\section{Results}

Pathogenic isolates of $M$. bovis differed in their capacity to grow in the cultured macrophages

To investigate the mechanisms employed by pathogenic Mbv to modulate $М \Phi$ activation, we selected for this 
study two clinical isolates of Mbv which showed significant difference in capacity of bacteria to grow in $М Ф$. As shown in Figure 1A, growth kinetics of one of the Mbv isolates, strain B2, was similar to that of the reference Mtb strain H37Rv. In contrast, the Mbv strain MP287/03 grew in MФ significantly faster $(\mathrm{p}<0.001)$.
After six days of incubation, an increase in the numbers of intracellular bacteria was 3-fold higher in cultures infected by the strain MP287/03, than those infected by strain B2. In contrast to the intracellular growth, growth rate of the tested strains in specific Middlebrook $7 \mathrm{H} 9$ media was similar, demonstrating that the intrinsic

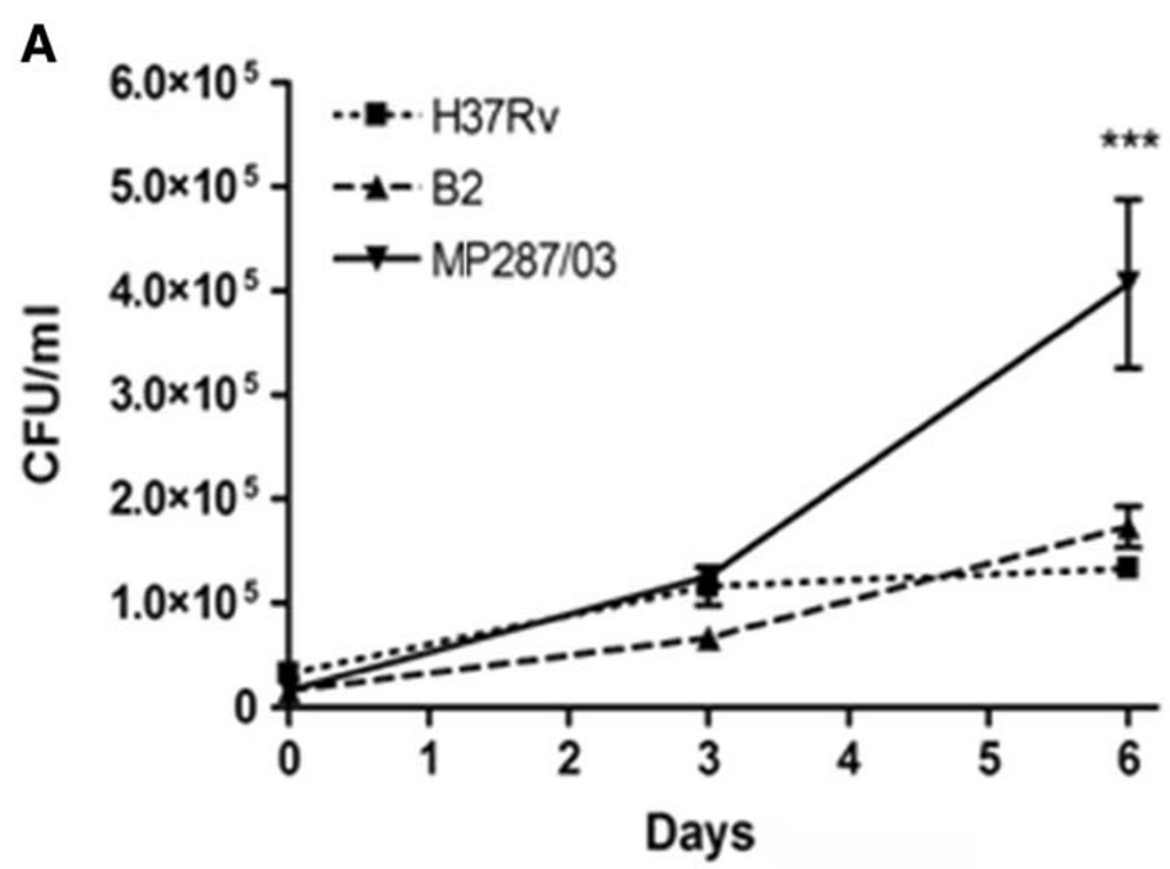

B

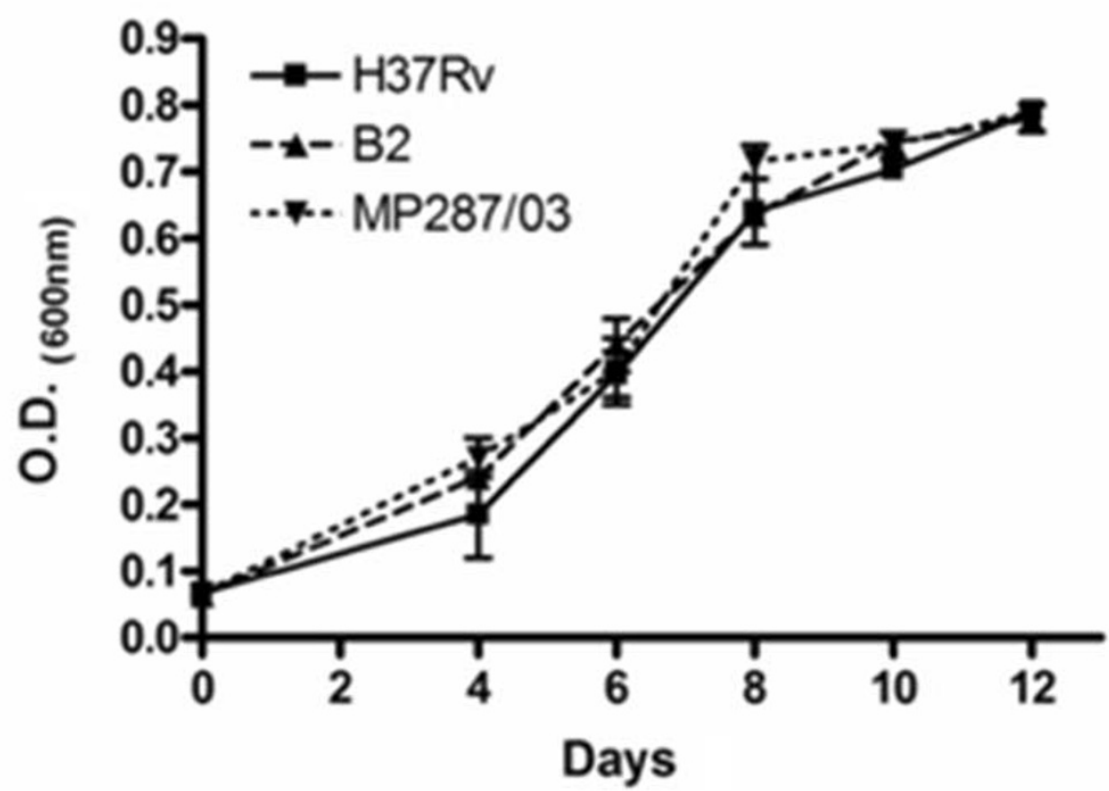

Figure 1 Evaluation of the growth properties of $\boldsymbol{M}$. bovis isolates. Isolates obtained from animals with tuberculosis, strains MP287/03 and B2, and reference M. tuberculosis strain H37Rv, were used for infection of BMDM in vitro (A) or cultured in Middlebrook $7 \mathrm{H} 9$ broth (B). Growth rates of mycobacteria inside $\mathrm{M} \Phi$ infected at $\mathrm{MOI}$ of 1 were determined using the colony count method. Intracellular CFU numbers were quantified immediately after infection (day 0) or at 3 or 6 days after infection (A). Growth rates of mycobacteria in 7 H9 Middlebrook broth were monitored by measurement of OD of the mycobacterial cultures by spectrophotometry. The growth curves of the mycobacterial strains within a 12 day period of incubation are presented. (B). Values are the means \pm SD of three independent experiments with samples in triplicate. 
abilities of the different strains to replicate were similar (Figure 1B). These data suggested that the observed differences in intracellular growth of these bacteria could be associated with differential resistance of the bacterial strains to microbicidal effects of $М \Phi$.

The main cytokines regulating proinflammatory $\mathrm{M} \Phi$ activity, IFN- $\gamma$ [16] and IL-10 [17], are known to increase or decrease the bactericidal functions of these cells, respectively. To verify whether intracellular survival of the different mycobacterial strains are equally regulated by the effects of IFN- $\gamma$ and IL-10 on MФ, we tested intracellular growth rates of the studied bacterial strains in BMDM cultured in the presence of these cytokines. As shown in Figure 2, the treatment of macrophage cultures with recombinant IL-10 had no significant effect on the growth of the studied strains. Treatment with IFN- $\gamma$ significantly reduced the growth rate of the strains $\mathrm{B} 2$ and $\mathrm{H} 37 \mathrm{Rv}$, but this effect was less pronounced in the cell cultures infected with the strain MP287/03. These data demonstrated the resistance of rapidly growing strain (MP287/03) to the effects of IFN$\gamma$, which is probably mediated by the ability of the mycobacteria to inhibit classic macrophage activation induced by this cytokine.

\section{Innate macrophage activation by the pathogenic} mycobacterial strains differing in growth kinetics in macrophages

To study the effects of pathogenic Mbv isolates on MФ activation, we evaluated characteristic markers of M1- and M2- type macrophage polarization induced in infected BMDM, in the presence or absence of IFN- $\gamma$ and IL-10.

First, we investigated the innate $M \Phi$ activation induced by infection. Evaluation of expression of the M1 proinflammatory markers, including factors mediating recruitment of the phagocytic cells (MCP-1/CCL2 and MIP-2/CXCL2), and contributing to the MФ microbicidity (TNF- $\alpha$, IL-12, IL-6 and NO), demonstrated that the studied pathogenic mycobacterial strains induced different patterns of cytokine secretion by the BMDM (Figure 3A). Both clinical isolates of $\mathrm{Mbv}$ induced less IL-6 and MCP-1, and, additionally, the Mbv strain MP287/03 induced less TNF- $\alpha$, than the reference strain H37Rv. In contrast, the level of secretion of MIP-2, an important chemokine regulating migration of granulocytes, was significantly increased in cultures infected with the Mbv strains. These cells secreted 10-fold more MIP-2 than the cells infected by H37Rv strain, and 3fold more than those infected by the strain B2. Neither mycobacterial strain tested in this study was able to induce in $M \Phi$ the production of NO or IL-12, although production of these mediators was induced by the LPS (Figure 3A).

Evaluation of the expression of typical M2 markers (IL-10, Arg-1 and MR/CD206) by the infected cells demonstrated that neither strain induced production of the IL-10 (Figure 3B). In contrast, all the studied mycobacterial strains were able to induce expression of Arg-1, and the highest level was observed in the cells infected with the strain MP287/03 (Figure 3C). The expression of MR, which was constitutively high in the intact uninfected BMDM, was suppressed by treatment of the cells with LPS, or infection with the less virulent $\mathrm{H} 37 \mathrm{Rv}$ and B2, whereas the cells infected with the strain MP287/03 continued to express high level of this receptor (Figure $3 \mathrm{C}$ ).

These data demonstrated that the proinflammatory activation of $\mathrm{M} \Phi$ by clinical isolates of $\mathrm{Mbv}$, and particularly by the fast growing strain MP287/03, was significantly

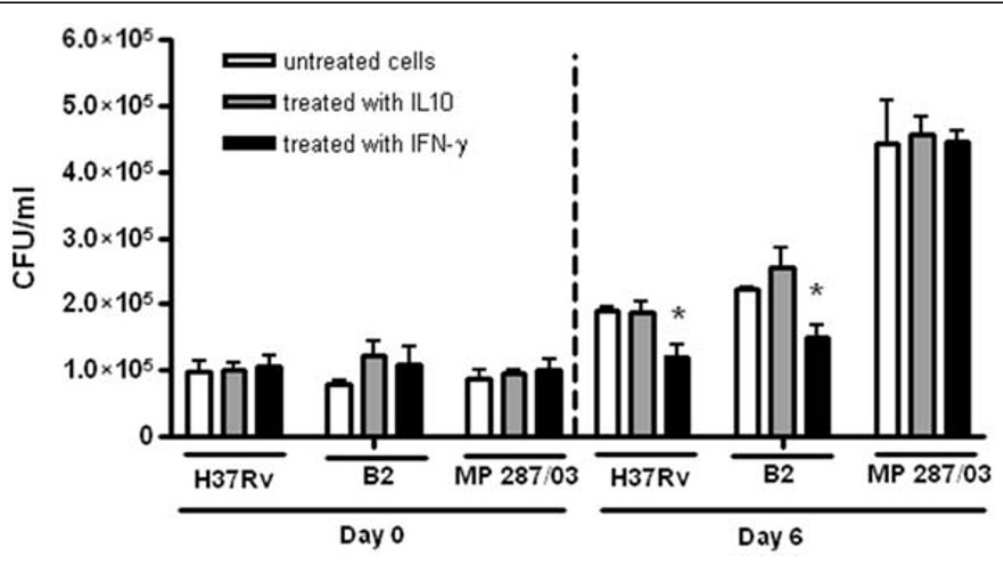

Figure 2 The capacity of pathogenic mycobacteria to grow intracellularly in macrophages treated with IFN- $\gamma$ or IL-10. Cultures of BMDM were pretreated with exogenic murine $\mathrm{r}-\mathrm{IFN}-\mathrm{\gamma}$ or $\mathrm{r}-\mathrm{IL}-10$ for $2 \mathrm{~h}$, infected with the mycobacterial strains at a $\mathrm{MOI}$ of 1 , as indicated in the legend to Figure 1, and incubated in the presence of these cytokines for an additional 6 days. The intracellular CFU numbers determined at day 0 and day 6 are presented. The data of three independent experiments are shown as mean \pm SD of samples in triplicate. Asterisks represent statistical significance $(p<0.05)$ compared to infected cells cultured without addition of the cytokines. 
A
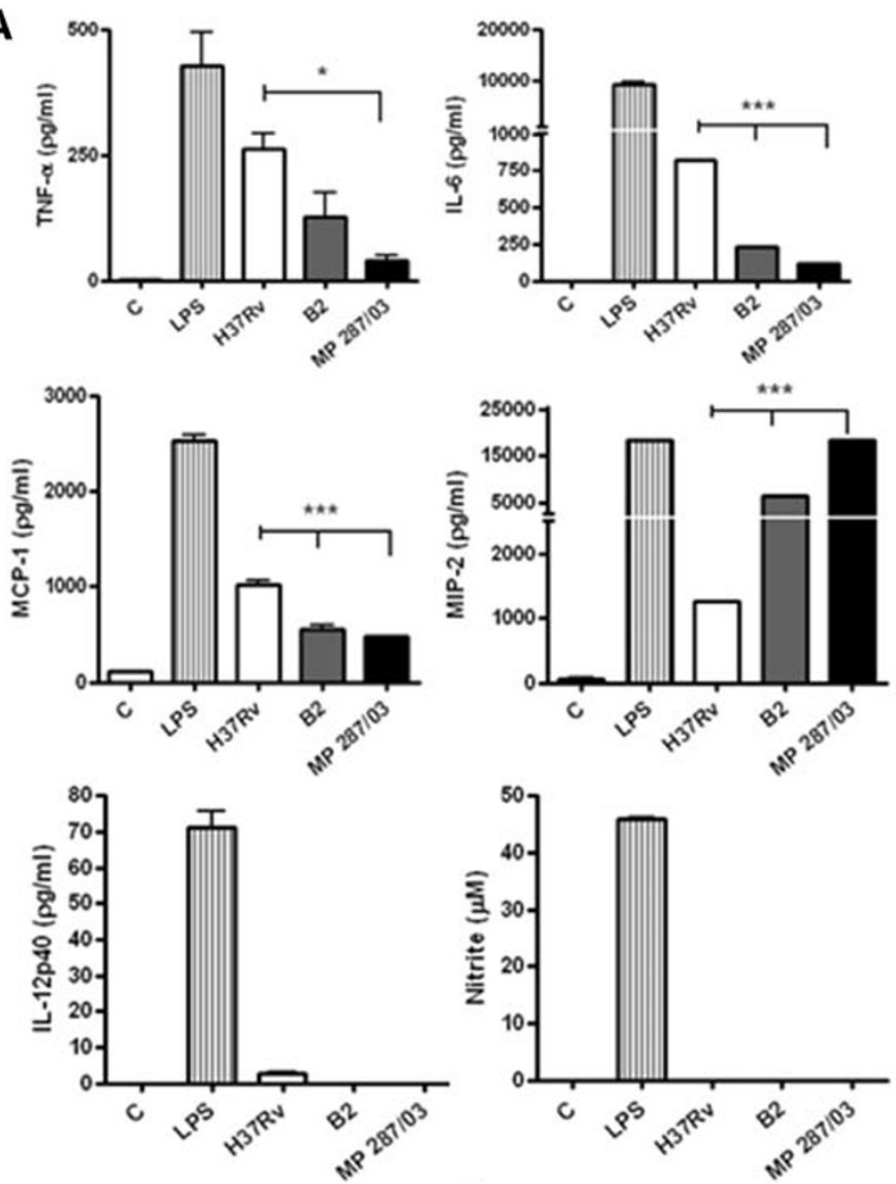

B

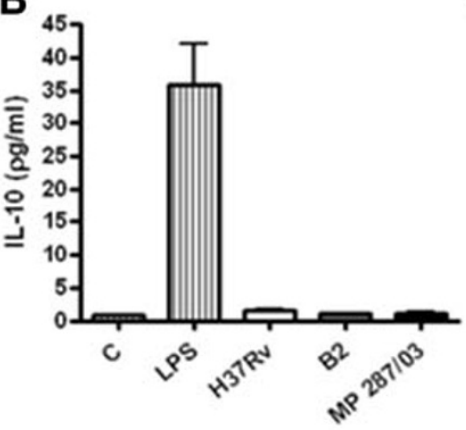

C

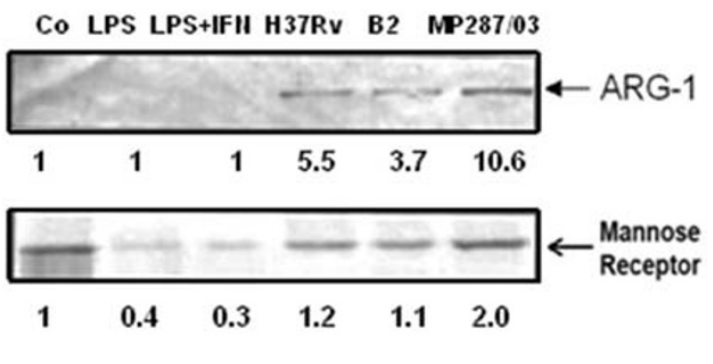

Figure 3 The activation profiles of macrophages infected with pathogenic mycobacteria. BMDM were infected with the studied mycobacterial strains at a MOI of 5:1, washed and incubated for an additional $\mathbf{4 8} \mathbf{h}$. The cells left untreated and cells stimulated with LPS for $48 \mathrm{~h}$ were used as a negative and positive controls of proinflammatory activation, respectively. To evaluate markers of M1-type activation (A), the culture supernatants of infected cultures were harvested and tested for TNF-a, IL-6, MCP-1, MIP-2 and IL-12 by Bioplex test, and for NO production by Griess reaction. Assays were completed with duplicate samples, and results are expressed as a mean of three independent experiments. Lines over bars indicate the isolates for which the induced cytokine production differed significantly from that induced by H37Rv $\left({ }^{*} p<0.05 ;{ }^{* *} p<0.001\right)$. To evaluate markers of M2-type activation, secretion of IL-10 was quantified by Bioplex assay (B), and expression of Arginase 1 and MR/CD206 in the adhered cells was tested by Western blotting (C). Lower panel, quantification of the protein levels by densitometric analysis of immunoreactive bands.

lower than that induced by the LPS or reference Mtb mycobacteria. Additionally, the strain MP287/03 induced in the $\mathrm{M} \Phi$ a more pronounced expression of some M2 markers.
However, strong secretion of proinflammatory MIP-2 chemokine observed in cell cultures infected by the strain MP287/03 suggested that these bacteria induced in MФ an atypical, mixed M1/M2 activation phenotype. 


\section{Modulating effects of the pathogenic mycobacterial strains on the macrophage activation phenotypes induced by the cell treatment with IFN- $\gamma$ and IL-10}

To study the MФ activation phenotypes resulted from combining effects of bacteria and regulating cytokines, we evaluated expression of the markers of M1 (Figure 4A-4D) and M2 cells (Figure 4E and 4F), by the pretreatment of infected BMDM with IFN- $\gamma$ (Figure 4A), and IL-10 (Figure 4B). The markers expressed by the infected cells, which were treated with the cytokines, were compared with those of the infected cells, which were left untreated. Treatment with IFN- $\gamma$ enhanced production of proinflammatory mediators in cultures infected by all the strains studied. However, the levels of secretion varied in a strain-dependent manner. Macrophages infected by the Mbv strains in the presence of IFN- $\gamma$ (Figure 4A) secreted significantly less TNF- $\alpha$, IL-6 and MCP-1, than those infected by the H37Rv strain. In contrast, production of MIP-2 by the cells infected with Mbv was significantly higher. As expected, treatment with IFN- $\gamma$ induced in the infected $M \Phi$, or those treated with LPS, production of NO (Figure 4A), which is an important mediator of $\mathrm{M} \Phi$ microbicidity, tightly regulated by the IFN- $\gamma$-dependent intracellular pathways. However, the cells infected with virulent Mbv strain B2, and particularly by the strain MP287/03, secreted significantly less NO than those infected with the H37Rv strain.

To verify whether signaling pathways leading to NO production were differentially modulated by the mycobacterial strains, we evaluated induction of iNOS, the essential enzyme for the conversion of arginine to citrulline and NO. The results obtained showed that treatment with IFN- $\gamma$ induced iNOS expression in the cultured macrophages, and subsequent infection of these cells with bacteria enhanced the level of enzyme expression in a similar manner (Figure 4D), demonstrating no strain-specific difference in the regulation of IFN- $\gamma$ dependent signaling which leads to transactivation of the iNOS gene.

Evaluation of expression of M2 markers in the cells pretreated with IFN- $\gamma$ demonstrated suppression of Arg1 expression induced by the strains B2 and H37Rv, but not those infected with strain MP287/03 (Figure 4E). Expression of MR by MФ was slightly inhibited in the cell cultures treated with IFN- $\gamma$, and further reduced after infection of these cells with the strains B2 or H37Rv. In contrast, infection with the strain MP287/03 restored a high level of expression of this receptor (Figure 4E), suggesting induction of MR gene transcription due to mycobacteria in these cells. Neither bacterial strain was able to induce IL-10 in the cells pretreated with the IFN- $\gamma$ (Figure 4F).

To verify the effects of mycobacterial infection on the IL-10-induced M2 polarization, the cell cultures were treated with recombinant IL-10. This treatment induced in the BMDM expression of Arg-1 (Figure 4E) and secretion of IL-10 (Figure 4F) and MCP-1 (Figure 4B). Infection of these cells with the mycobacterial strains promoted expression of M2 markers, further increasing expression of the Arg-1 and suppressing inhibition of the MR expression induced by the H37Rv and B2 strains (Figure 4E). The infected cultures continued to secrete low levels of IL-10, induced by the exogenic IL-10 pretreatment (Figure 4F). Additionally, the treatment of $M \Phi$ with IL-10 suppressed ability of some mycobacterial strains to induce increased levels of secretion of proinflammatory mediators. Significant reduction of secretion of IL- 6 and MCP- 1 by MФ infected with the H37Rv strain and MIP-2 chemokine secretion, induced by the strains B2 and MP287/03, was observed (Figure 4B).

These data show that the proinflammatory activities of $M \Phi$ induced by mycobacterial infection were significantly inhibited in the cells that were infected after priming by IL-10. These cells expressed MR and increased levels of Arg-1, which were particularly high in the cells infected with MP287/03 strain. Thus, the treatment with IL-10 favored M2-type activation of the infected $M \Phi$.

\section{Discussion}

In this study, we aimed to investigate the modulating effects of pathogenic Mbv strains, differing in virulenceassociated properties, on activation phenotypes in MФ treated with the main cytokines regulating proinflammatory $M \Phi$ activation: IFN- $\gamma$ and IL-10. Rapid growth of pathogenic mycobacteria in $M \Phi$ is one of the known factors contributing to bacterial virulence $[18,19]$. Therefore, for this work, we selected two Mbv isolates differing significantly in the capacity to grow in $М Ф$. One of these isolates, strain B2, was capable of growing in $\mathrm{BMDM}$ at a rate similar to that of moderately virulent Mtb strain H37Rv, whereas the intracellular multiplication of other Mbv strain (MP287/03) was significantly faster. Additionally, we demonstrated that bacteria of MP287/03 strain continued to grow rapidly in cells activated by IFN- $\gamma$, whereas the growth of the strains B2 and $\mathrm{H} 37 \mathrm{Rv}$ was significantly inhibited under this treatment. These data suggested that the MP287/03 strain was either more resistant to the bactericidal effects of macrophages classically activated by IFN- $\gamma$, or were able to inhibit $\mathrm{M} \Phi$ activation induced by this cytokine.

The modulating effects of the Mbv strains were evaluated in comparison to those of the reference Mtb strain $\mathrm{H} 37 \mathrm{Rv}$, which was demonstrated in previous studies to induce in $\mathrm{M} \Phi$ a proinflammatory activation and synergize with IFN- $\gamma$ in induction of M1-type polarization of infected cells $[7,20]$. In accordance with these observations, our data demonstrated that the Mtb strain H37Rv 

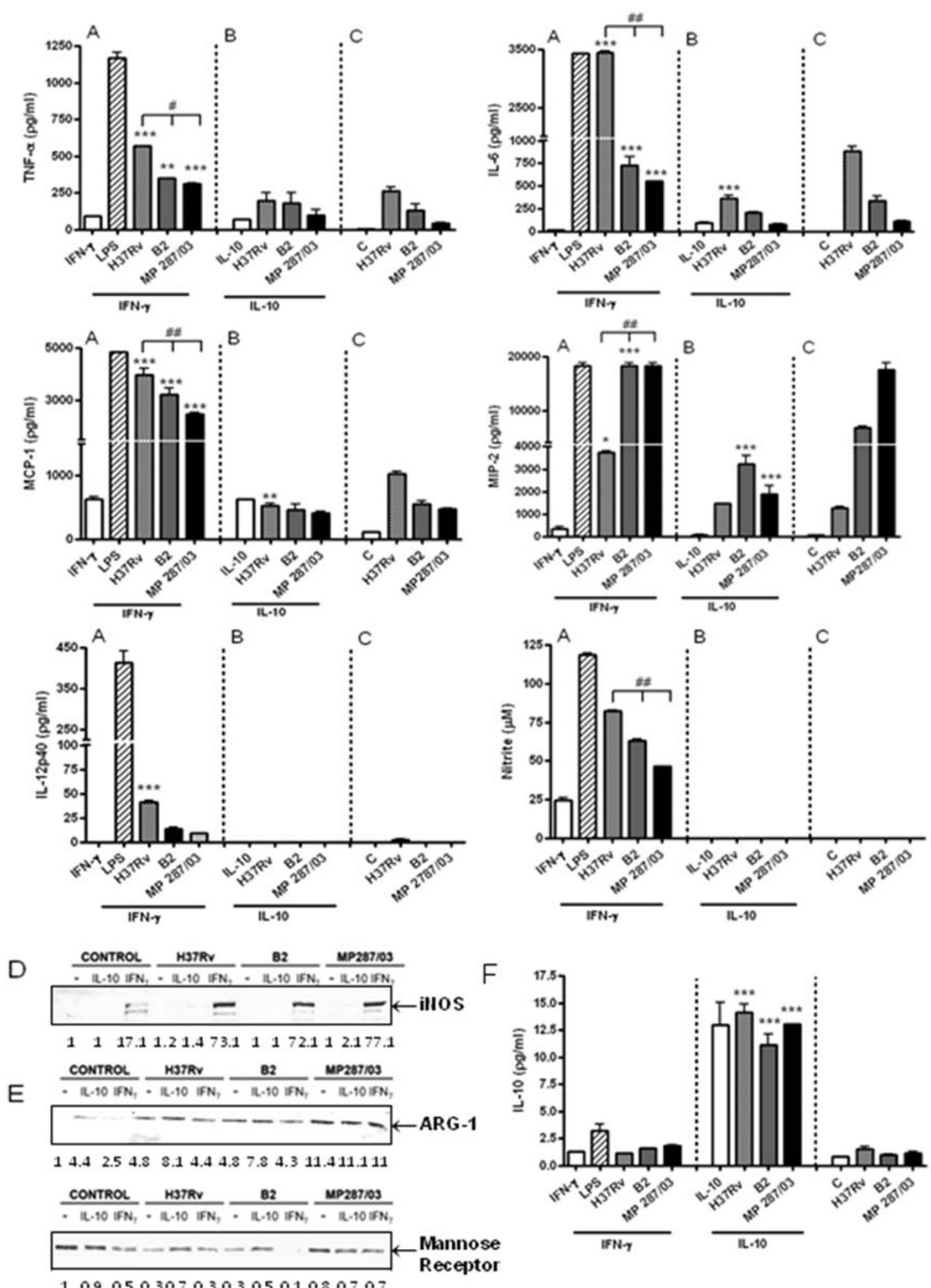

Figure 4 (See legend on next page.) 
(See figure on previous page.)

Figure 4 The activation profiles of macrophages treated with IFN- $\gamma$ or IL-10 and infected with pathogenic mycobacteria. BMDM were pretreated, or not, with murine $r-I F N-\gamma$ or $r-I L-10$ for $2 \mathrm{~h}$, infected with the studied mycobacterial strains at a MOI of 5:1, washed, treated again with the cytokines and incubated for an additional $48 \mathrm{~h}$. The cells stimulated with LPS and r-IFN- $\gamma$ for $48 \mathrm{~h}$, or left untreated, were used as a positive and negative controls of classical proinflammatory activation, respectively. To evaluate markers of M1-type activation, the culture supernatants were tested for proinflammatory mediator levels (A-C) and the adhered cells were tested for expression of iNOS (D). Measurement of TNF-a, IL-6, MCP-1, MIP-2 and IL-12 concentrations was performed by Bioplex test, and NO production was evaluated by Griess reaction Assays were completed with duplicate samples, and results are expressed as a mean of three independent experiments. To evaluate markers of M2-type activation, expression of Arginase 1 and MR/CD206 in the adhered cells was tested by Western blotting (E) and secretion of IL-10 was quantified by Bioplex assay (F). Lower panels in $D$ and $E$, quantification of the protein levels by densitometric analysis of immunoreactive bands. Asterisks in $A, B$ and $F$ indicate the infected cultures treated with recombinant IFN- $\gamma$ or IL-10, for which the induced cytokine production differed significantly from that in the corresponding cultures incubated without the presence of exogenic cytokines $\left(^{*} p<0.05 ;{ }^{* *} p<0.01 ;{ }^{* *} p<0.001\right)$. Lines over bars in $\mathrm{A}$ and $\mathrm{B}$ indicate the $\mathrm{Mbv}$ isolates for which the induced cytokine or $\mathrm{NO}$ production differed significantly from that induced by $\mathrm{H} 37 \mathrm{RV}$

$(\# p<0.01 ; \# \#<0.001)$.

induced M1 type activation of the infected BMDM, although the level of activation was less pronounced in comparison with that induced by LPS. Activation of MФ by the Mbv strains was even weaker than that induced by the H37Rv strain. The lowest level of proinflammatory cytokine expression was observed in $M \Phi$ infected with the fast growing Mbv strain MP287/03, although these cells produced high levels of MIP-2 chemokine. Additionally, these cells displayed increased levels of expression of M2 markers (Arg-1 and MR/CD206). Thus, the MP287/03 mycobacteria induced in MФ an atypical, mixed M1/M2 activation phenotype that coincided with enhanced intracellular growth of the bacteria.

Most important was observation, that this strain induced weaker production of the key bactericidal factors, such as TNF- $\alpha$ and NO, even after pretreatment of $M \Phi$ with IFN- $\gamma$, priming these cells for M1-type activation. To study the mechanisms that could underlie the observed differences in RNI production, we looked at intracellular signaling pathways leading to NO production by the infected cells. The major regulators of $\mathrm{NO}$ production are iNOS and Arg -1, competitive enzymes which utilize a common substrate (L-arginine) to produce NO and citrulline, or urea and ornithine, respectively [21]. In previous study [22], induction of Arg-1 expression in $M \Phi$ by attenuated Mbv strain BCG was found to be essential for reduction of NO production, through the arginine substrate depletion mechanism, leading to promotion of the intracellular survival of these mycobacteria.

In this study, we demonstrated that pathogenic Mbv were also able to induce expression of Arg-1 in the infected $M \Phi$. Importantly, the fast growing strain MP287/03 induced higher levels of the Arg-1, than any other studied strain, and strongly up-regulated expression of Arg-1 in IFN- $\gamma$-treated cells. Although all of the studied strains enhanced expression of iNOS, induced in cells by IFN- $\gamma$, in a similar manner, the increased level of Arg-1 observed in MФ infected with the MP287/03 strain contributed to reduction of $\mathrm{NO}$ secretion by these cells. These data suggested that highly virulent Mbv, characterized by enhanced growth in $М \Phi$ could induce Arg-1 as a component of the strategy to subvert the antimicrobial activity of CAM, by hydrolyzing the substrate required for $\mathrm{NO}$ production.

Mechanisms leading to induction of Arg-1 expression by mycobacteria are only recently starting to be elucidated. Autocrine loop of secretion of IL-6, IL-10 and GCSF, leading to phosphorylation of STAT3 was determined as an essential mechanism for induction of Arg-1 expression in BCG-infected MФ [22]. However, in our study, the increased Arg-1 expression induced by the strain MP287/03, coincided with low levels of IL-6 and IL-10 secretion by the infected MФ. These data suggested that the signaling pathways, leading to the pronounced induction of the Arg-1 by highly virulent Mbv, could differ from those induced in the BCG-infected $M \Phi$ and should be investigated further in separate study.

Another mechanism, underlying the reduced microbicidity of MФ infected with the strain MP287/03, could be associated with capacity of these bacteria to induce in the cells expression of MR. Infection with the strain $\mathrm{H} 37 \mathrm{Rv}$ and incubation with IFN- $\gamma$, synergistically inhibited expression of MR gene in murine BMDM [7,23], constitutively expressing high levels of MR [23], resembling in this manner, alveolar macrophages [24]. In line with these observations, infection of the cells pretreated with IFN $-\gamma$ by the moderately virulent strains, H37Rv and B2, in our experiments resulted in down-regulation of MR expression. In contrast to these strains, infection of MФ by the strain MP287/03 restored expression of MR reduced by the IFN- $\gamma$ treatment. High and persistent levels of MR expression in the MФ infected with strain MP287/03 in the presence or absence of IFN- $\gamma$ suggested that these cells could be more susceptible to the deleterious effects of Mannosyl-capped lipoarabinomannan (ManLAM) expressed by the pathogenic 
mycobacteria. Interaction of Man-LAM with MR has been demonstrated to inhibit fusion of phagosomes with lysosomes in the infected $M \Phi$, interfere with IFN- $\gamma$ mediated signaling in $M \Phi$ activation, as well as suppress TLR-dependent induction of expression of IL-12 and other proinflammatory cytokines [25,26]. In line with this suggestion, the infected cells expressing higher levels of $\mathrm{MR}$ in our experiments were permissive to enhanced intracellular growth even in the presence of IFN- $\gamma$.

The ability of the strain MP287/03 to induce in MФ some properties of the M2 cells, suggested that infection of the $M \Phi$, pretreated with IL-10, by these bacteria may synergize in IL-10- dependent M2 polarization of these cells. The obtained results demonstrated that the treatment with IL-10 led to reduction of the proinflammatory $M \Phi$ activation by the studied mycobacterial strains. These cells displayed increased expression of the M2 markers, MR, IL-10 and Arg-1. The highest levels of Arg-1 were observed in the cells infected by MP287/03 mycobacteria, demonstrating that the treatment with IL10 favored the M2-type activation of these cells.

Although the cells infected with MP287/03 strain displayed increased levels of the M2 markers in the presence or absence of regulating cytokines, these cells secreted high levels of the proinflammatory MIP-2 chemokine. In contrast to the $\mathrm{MCP}-1$ chemokine, regulating monocyte recruitment which is essential for formation of functional granuloma, the continues production of MIP-2, and other chemokines attracting granulocytes, was demonstrated to cause excessive recruitment of neutrophils to the infected lungs, contributing to tissue damage in pulmonary tuberculosis, reviewed by [27]. The high level of MIP-2 secretion and inappropriate proinflammatory $M \Phi$ activation, observed in the BMDM cultures infected with MP287/03 strain in this study, may have aggravating implications for in vivo infection with these, fast-replicating intracellular bacteria. Verification of this important issue is currently under investigation in our laboratory.

\section{Conclusions}

The data obtained in this study show that the pathogenic Mbv strains differed in their capacity to modulate the M1type activation phenotype induced by IFN- $\gamma$. In contrast to the mycobacterial strains demonstrating moderate ability to grow intracellularly which enhanced classical activation of MФ by INF- $\gamma$, the fast growing strain of $\mathrm{Mbv}$ induced an atypical, mixed M1/M2 phenotype, leading to inhibition of MФ bactericidal activity. These data demonstrate functional diversity of Mbv strains circulating in animal population, highlighting novel strategies of intracellular adaptation of the pathogenic mycobacteria. Elucidating the functional significance of diversity of virulence-associated properties of $\mathrm{Mbv}$ is important for understanding the diverse outcomes of infection and mechanisms of pathogenesis of bovine tuberculosis.

\section{Methods \\ Mycobacteria}

Two isolates of $\mathrm{Mbv}$ from animals with tuberculosis were used in this study. The strain B2 was isolated from buffalo and gently provided by Dr. Eliana Roxo (Biological Institute, USP, São Paulo, Brazil). The bovine strain MP287/03 was kindly provided by Dr. José Soares Ferreira Neto (Institute for Veterinary Medicine, USP, São Paulo, Brazil). M. tuberculosis strain H37Rv (ATCC) was kindly provided by Dr. Philip Suffys (Oswaldo Cruz Foundation, FIOCRUZ, Rio de Janeiro, Brazil).

Mycobacterial strains were grown in suspension in complete 7H9 Middlebrook broth (Difco, Detroit, MI), containing 10\% albumin dextrose complex, ADC (BD, Sparks, MD), $0.5 \%$ glycerol and $0.05 \%$ Tween- 80 at $37^{\circ} \mathrm{C}$ under Biosecurity level 3 containment conditions. Additionally, sodium pyruvate $0.4 \%$ was added to the cultures of Mbv. Bacterial cultures were grown to midlogarithmic phase, aliquoted, and stored at $-70^{\circ} \mathrm{C}$. Before experiments, the aliquots were thawed, resuspended in complete 7H9 medium and cultured for 5 days. Bacterial suspensions were ultrasonicated in water bath to disrupt small clumps and obtain single cell suspensions. The resulted dispersion of bacteria was tested by microscopic examination of the suspension samples stained by the acid-fast staining procedure. The densities of the suspensions were measured by spectrophotometry, and corresponding concentrations were determined by serial dilution plating of each strain on Middlebrook $7 \mathrm{H} 10$ agar (Difco, Detroit, MI) plates supplemented with $0.5 \%$ glycerol, $10 \%$ oleic acid-albumin-dextrose-catalase enrichment, OADC (BD, Sparks, MD), and, additionally, with $0.4 \%$ sodium pyruvate in the case of Mbv cultures. After 21 days, total CFU were determined.

\section{Quantification of mycobacterial growth in $7 \mathrm{H} 9$ broth}

The bacterial capacity to grow in 7H9 broth was measured by spectrophotometry. Bacterial suspensions adjusted to $\mathrm{OD}_{600}=0.1$ were cultured at $37^{\circ} \mathrm{C}$ for twelve days with daily agitation. Bacterial tubes were then vortexed, ultrasonicated in a water bath, and the OD of suspension was measured. To confirm the lack of significant alteration in $\mathrm{OD}_{600}$ readings, colony forming units (CFU) were determined for each culture on day 0 through the plating of appropriate bacterial dilution onto the 7H10 agar.

\section{Generation of bone marrow- derived macrophages}

Bone marrow- derived macrophages (BMDM) were obtained as previously described [28] with some modifications. Briefly, bone marrow cells were flushed from 
the femur of eight- to ten- week-old specific pathogenfree C57BL/6 mice. The cells were dispersed in Dulbecco's modified Eagle's medium, DMEM (Sigma, St Louis, MO), supplemented with $1 \mathrm{mM}$ sodium pyruvate, $2 \mathrm{mM}$ L-glutamine, 0.05 M 2-mercaptoethanol (Gibco BRL, Grand Island, NY), 10\% heat-inactivated FBS (Hyclone, Road Logan, UT), $50 \mu \mathrm{g} / \mathrm{ml}$ gentamicin (Gibco BRL, Grand Island, NY), and cultured at $37^{\circ} \mathrm{C}$ in $5 \% \mathrm{CO}_{2}$ atmosphere. Nonadherent cells were collected after $18 \mathrm{~h}$, resuspended in the complete DMEM, supplemented with 20\% L929 cellconditioned medium as a source of M-CSF, and cultured for 7 days, replacing the medium on day 3. The monolayer cells were scraped, resuspended in DMEM, supplemented with $2 \% \mathrm{FBS}$, without antibiotics, and plated at a concentration of $5 \times 10^{5} \mathrm{cell} / \mathrm{ml}$ in a 96-well plates, $100 \mu \mathrm{l} /$ well.

\section{Treatment with cytokines and infection of cell cultures}

The BMDM cultures were incubated overnight, pretreated, or not, with murine recombinant IFN- $\gamma, 100 \mathrm{U} / \mathrm{ml}$ (Bioscience, Camarillo, CA), or IL-10, 20 ๆg/ml (Bioscience, Camarillo, CA) for $2 \mathrm{~h}$, and infected with single-cell suspensions of mycobacterial strains at MOI 1:1 and 5:1. After $3 \mathrm{~h}$ of incubation at $37^{\circ} \mathrm{C}$, infected monolayers were washed and incubated for additional $6 \mathrm{~d}$ in new aliquots of culture medium. In the pretreated cultures, the cytokines were renewed and were present throughout the incubation period. Cell viability of infected MФ was monitored by trypan blue exclusion and was over $90 \%$ in all experiments.

\section{Quantification of mycobacterial growth in macrophages}

Mycobacterial ability to grow intracellularly was evaluated by colony-forming units (CFU) test in the MФ cultures infected at a MOI of 1:1. After 0,3 and $6 \mathrm{~d}$ of incubation, cells were lysed with $1 \%$ saponin to release intracellular bacteria. Lysates of infected cells were resuspended, transferred into screw caps, vortexed and sonicated in a preheated waterbath sonicator (Unique 800 , Brazil) at $37^{\circ} \mathrm{C}$ for $2 \mathrm{~min}$. Aliquots of the sonicate were diluted 10 -fold in PBS, plated in quadriplicates on $7 \mathrm{H} 10$ agar plates and incubated at $37^{\circ} \mathrm{C}$ for 21 days.

\section{Cytokine quantification}

To study cytokines secreted by infected $M \Phi$, the cell cultures were infected at a MOI 5:1 in the presence or absence of recombinant IFN- $\gamma$ and IL-10, as indicated above. The infected monolayers were washed and incubated for additional $48 \mathrm{~h}$. After incubation, the culture supernatants were collected, filtered through $0.22 \mu \mathrm{m}$ Spin-X centrifuge tube filters (Corning, NY), and the supernatant aliquots were stocked at $-70^{\circ} \mathrm{C}$ for posterior cytokine determination. The cells left untreated and cells stimulated with LPS of Escherichia coli 011B (Sigma Aldrich, MO), $1 \mu \mathrm{g} / \mathrm{ml}$, and $\mathrm{r}$-IFN- $\gamma$ for $48 \mathrm{~h}$ were used as a negative and positive control of proinflammatory macrophage activation, respectively. The frozen samples of culture supernatants of the infected BMDM were then thawed and immediately analyzed using Bio-Plex Pro Mouse Cytokine Assay (BioRad Laboratories, Hercules, CA), following the manufacturers protocol. Standard curves for each cytokine were generated using reference cytokine concentrations supplied by the manufacturer.

\section{Nitric oxide determination}

Nitric oxide (NO) generation in the culture supernatants was assessed by the Griess method to measure nitrites, which are stable breakdown products of NO. Briefly, culture supernatant was incubated with the Griess reagents I (1\% sulfanilamide in $2.5 \%$ phosphoric acid) and II ( $0.1 \%$ naphthylenediamine in $2.5 \%$ phosphoric acid). The absorbency was read within $5 \mathrm{~min}$ at $550 \mathrm{~nm}$ and actual concentration calculated using a standard curve with serial dilutions of sodium nitrite.

\section{Detection of iNOS, ARG-1 and MR by Western blot}

The infected adherent cells were resuspended in lysis buffer (10\% SDS, 20\% glycerol, 5\% 2-mercaptoethanol, $2 \%$ bromphenol blue and $1 \mathrm{M}$ Tris $\mathrm{HCl}, \mathrm{pH}$ 6.8) for western blotting analysis. Cell samples in the lysis buffer were harvested and equal amounts of proteins were electrophoresed in a $10 \%$ or $8 \%$ sodium SDS-PAGE gel under nonreducing conditions. The proteins were then transferred to nitrocellulose membrane (Amersham Hybond-ECL GE) using standard procedures. After overnight blocking with $0.5 \%$ non-fatty milk in PBS, the blots were incubated for $1 \mathrm{hr}$ at room temperature with $\mathrm{Ab}$ against iNOS, 1:1000 (Santa Cruz Biotechnology, CA), Arg-1, 1:1000 (BD Bioscience), or MR/CD206, 1:100 (Santa Cruz Biotechnology, CA), dissolved in 0.5\% nonfatty milk in PBS. The blots were then washed and incubated with peroxidase-conjugated secondary Ab, 1:8000, for $1 \mathrm{hr}$ at room temperature, and the resulting membranes were developed using diaminobenzidine $/ \mathrm{H}_{2} \mathrm{O}_{2}$ as a substrate for peroxidase. Densitometric analysis of the protein bands was performed using the software ImageJ for Windows (NIH, Bethesda, MD). The value for the control condition (untreated cells) was set as 1 and other conditions were recalculated correspondingly to allow ratio comparisons.

\section{Statistical analysis}

Statistical analysis was performed using the unpaired Student's $t$ test, one-way analysis of variance (ANOVA) and Bonferroni procedure for multiple range tests, employing Prism 4 software (GraphPad, San Diego, CA) to assess statistical significance between groups of data 
defining different error probabilities. A value of $\mathrm{p}<0.05$ was considered to be significant.

\section{Abbreviations}

AAM: Alternatively Activated Macrophages; Arg-1: Arginase 1; BMDM: Bone Marrow- Derived Macrophages; CAM: Classically Activated Macrophages; IFN-Y: Interferon gamma; IL-10: Interleukin 10; iNOS: Inducible Nitric Oxide Synthase; LPS: Lipopolysaccharide; MФ: Macrophages; Mbv: Mycobacterium bovis; Mtb: Mycobacterium tuberculosis; MR: Mannose Receptor; MCP1: Monocyte Chemoattracting Protein; MIP-2: Macrophage Inflammatory Protein 2; NO: Nitric Oxide; ROI: Reactive Oxygen Intermediates; RNI: Reactive Nitrogen Intermediates; TLR: Toll-like Receptors.

\section{Competing interests}

The authors declare that they have no competing interests.

\section{Authors' contributions}

MRMA performed the experiments and prepared the figures; EPA evaluated growth curves of mycobacteria in $M \Phi$ and broth; VL cultured and characterized the mycobacterial strains; TVP established the in vitro model of BMDM infection; EPA, SCMR and FMA carried out the immunoassays; EBL, MRIL and MRMA analyzed the data; EL and MRMA conceived of, designed the study and wrote the manuscript, MREL revised the manuscript critically. All authors read and approved the final manuscript.

\section{Acknowledgements}

This work was supported by Fundação de Amparo a Pesquisa de Rio de Janeiro (FAPERJ) and Conselho Nacional de Desenvolvimento Científico e Tecnológico (CNPq), Brazil.

\section{Author details}

'Laboratory of Biology of Recognition, Universidade Estadual do Norte Fluminense, Campos Rio de Janeiro 28013-602, Brazil. ${ }^{2}$ Department of Immunology, Universidade de São Paulo, São Paulo 05508-900, Brazil.

Received: 8 March 2012 Accepted: 18 July 2012

Published: 3 August 2012

\section{References}

1. Gordon S: Alternative activation of macrophages. Nat Rev Immunol 2003, 3:23-35.

2. Mantovani A, Sica A, Sozzani S, Allavena P, Vecchi A, Locati M: The chemokine system in diverse forms of macrophage activation and polarization. Trends Immunol 2004, 25:677-686.

3. Mosser DM, Edwards JP: Exploring the full spectrum of macrophage activation. Nat Rev Immunol 2008, 8:958-969.

4. Lopez-Castejón G, Baroja-Mazo A, Pelegrín P: Novel macrophage polarization model: from gene expression to identification of new anti-inflammatory molecules. Cell Mol Life Sci 2011, 68:3095-3107.

5. Kleinnijenhuis J, Oosting M, Joosten LA, Netea MG, Van Crevel R: Innate immune recognition of Mycobacterium tuberculosis. Clin Dev Immunol 2011, 201(1):405310.

6. Ehrt S, Schnappinger D, Bekiranov S, Drenkow J, Shi S, Gingeras TR, Gaasterland T, Schoolnik G, Nathan C: Reprogramming of the macrophage transcriptome in response to interferon- and Mycobacterium tuberculosis: signaling roles of nitric oxide synthase-2 and phagocyte oxidase. J Exp Med 2001, 194:1123-1140.

7. Kahnert A, Seiler P, Stein M, Bandermann S, Hahnke K, Mollenkopf H, Kaufmann SH: Alternative activation deprives macrophages of a coordinated defense program to Mycobacterium tuberculosis. Eur J Immunol 2006, 36:631-647.

8. Aguilar LD, Zumárraga MJ, Oropeza JR, Gioffré AK, Bernardelli A, Orozco EH Cataldi AA, Hernández P: Mycobacterium bovis with different genotypes and from different hosts induce dissimilar immunopathological lesions in a mouse model of tuberculosis. Clin Exp Immunol 2009, 57:139-147.

9. Meikle V, Bianco MV, Blanco FC, Gioffré A, Garbaccio S, Vagnoni L, Di Rienzo J, Canal A, Bigi F, Cataldi A: Evaluation of pathogenesis caused in cattle and guinea pig by a Mycobacterium bovis strain isolated from wild boar. BMC Vet Res 2011, 12:7-37.

10. López B, Aguilar D, Orozco H, Burger M, Espitia C, Ritacco V, Barrera L, Kremer K, Hernandez-Pando R, Huygen K, van Soolingen D: A marked difference in pathogenesis and immune response induced by different Mycobacterium tuberculosis genotypes. Clin Exp Immunol 2003, 133:30-37.

11. Ordway D, Henao-Tamayo M, Harton M, Palanisamy G, Troudt J, Shanley C, Basaraba RJ, Orme IM: The hypervirulent Mycobacterium tuberculosis strain HN878 induces a potent TH1 response followed by rapid down-regulation. J Immunol 2007, 179:522-531.

12. Park JS, Tamayo MH, Gonzalez-Juarrero M, Orme IM, Ordway DJ: Virulent clinical isolates of Mycobacterium tuberculosis grow rapidly and induce cellular necrosis but minimal apoptosis in murine macrophages. J Leukoc Biol 2006, 79:80-86.

13. Lasunskaia E, Ribeiro SC, Manicheva O, Gomes LL, Suffys PN, Mokrousov I, Ferrazoli L, Andrade MR, Kritski A, Otten T, Kipnis TL, da Silva WD, Vishnevsky B, Oliveira MM, Gomes HM, Baptista IF, Narvskaya O: Emerging multidrug resistant Mycobacterium tuberculosis strains of the Beijing genotype circulating in Russia express a pattern of biological properties associated with enhanced virulence. Microb Infect 2010, 12:467-475.

14. Rook GA, Steele J, Ainsworth M, Champion BR: Activation of macrophages to inhibit proliferation of Mycobacterium tuberculosis: comparison of the effects of recombinant gamma-interferon on human monocytes and murine peritoneal macrophages. Immunology 1986, 59:333-338.

15. Flesch I, Kaufmann SH: Mycobacterial growth inhibition by interferongamma -activated bone marrow macrophages and differential susceptibility among strains of Mycobacterium tuberculosis. J Immunol 1987, 138:4408-4413.

16. Nathan CF, Murray HW, Wiebe ME, Rubin BY: Identification of interferon-gamma as the lymphokine that activates human macrophage oxidative metabolism and antimicrobial activity. J Exp Med 1983, 158:670-689.

17. Lang R: Tuning of macrophage responses by STAT3-inducing cytokines: molecular mechanisms and consequences in infection. Immunobiology 2005, 210:63-76.

18. Silver RF, Li Q, Ellner JJ: Expression of virulence of Mycobacterium tuberculosis within human monocytes: virulence correlates with intracellular growth and induction of tumor necrosis factor alpha but not with evasion of lymphocyte-dependent monocyte effector functions. Infect Immun 1998, 66:1190-1199.

19. Lukey PT, Hooker EU: Mycobacterium tuberculosis protocols. In Macrophage Virulence Assays. Edited by Parish T, Stoker NG. New Jersey: Humana Press, Totowa; 2003.

20. Redente EF, Higgins DM, Dwyer-Nield LD, Orme IM, Gonzalez JM, Malkinson AM: Differential polarization of alveolar macrophages and bone marrow-derived monocytes following chemically and pathogen-induced chronic lung inflammation. J Leukoc Biol 2010 88:159-168.

21. Modolell M, Corraliza IM, Link F, Soler G, Eichmann K: Reciprocal regulation of the nitric oxide synthase/arginase balance in mouse bone marrow-derived macrophages by TH1 and TH2 cytokines. Eur J Immunol 1995, 25:1101-1104.

22. El Kasmi KC, Qualls JE, Pesce JT, Smith AM, Thompson RW, HenaoTamayo M, Basaraba RJ, König T, Schleicher U, Koo MS, Kaplan G, Fitzgerald KA, Tuomanen El, Orme IM, Kanneganti TD, Bogdan C, Wynn TA, Murray PJ: Toll-like receptor-induced arginase 1 in macrophages thwarts effective immunity against intracellular pathogens. Nat Immunol 2008, 9:1399-1406

23. Schreiber S, Perkins SL, Teitelbaum SL, Chappel J, Stahl PD, Blum JS: Regulation of mouse bone marrow macrophage mannose receptor expression and activation by prostaglandin E and IFN-gamma. $J$ Immunol 1993, 151:4973-4981.

24. Torrelles JB, Schlesinger LS: Diversity in Mycobacterium tuberculosis mannosylated cell wall determinants impacts adaptation to the host. Tuberculosis 2010, 90:84-93.

25. Nigou J, Zelle-Rieser C, Gilleron M, Thurnher M, Puzo G: Mannosylated lipoarabinomannans inhibit IL-12 production by human dendritic cells: evidence for a negative signal delivered through the mannose receptor. J Immunol 2001, 166:7477-7485.

26. Pathak SK, Basu S, Bhattacharyya A, Kundu M, Basu J: Mycobacterium tuberculosis lipoarabinomannan-mediated IRAK-M induction negatively regulates Toll-like receptor-dependent interleukin-12 p40 production in macrophages. J Biol Chem 2005, 280:42794-42800. 
27. Lowe DM, Redford PS, Wilkinson RJ, O'Garra A, Martineau AR: Neutrophils in tuberculosis: friend or foe? Trends Immunol 2012. 1:14-25.

28. Weischenfeldt J, Porse B: Bone Marrow-Derived Macrophages (BMM): Isolation and Applications. Cold Spring Harb Protoc 2008, doi:10.1101/ pdb.prot5080

doi:10.1186/1471-2180-12-166

Cite this article as: Andrade et al:: Pathogenic Mycobacterium bovis strains differ in their ability to modulate the proinflammatory activation phenotype of macrophages. BMC Microbiology 2012 12:166.

\section{Submit your next manuscript to BioMed Central and take full advantage of:}

- Convenient online submission

- Thorough peer review

- No space constraints or color figure charges

- Immediate publication on acceptance

- Inclusion in PubMed, CAS, Scopus and Google Scholar

- Research which is freely available for redistribution 